\title{
Feto-Maternal Outcome In Caesarean Sections Performed In Second Stage of Labour
}

Thirukumar $M^{1}$.

${ }^{1}$ Faculty of Health Care Science, Eastern University, Sri Lanka.

\section{Abstract}

The second stage caesarean section is associated increased maternal and foetal morbidity due to its inherent technical difficulties.

This was a prospective observational study of 25 cases of caesarean sections done in second stage of labour in Teaching Hospital Batticaloa, Sri Lanka. The main aim of the study was to identify the maternal and perinatal outcomes of second stage caesarean sections. The findings of this study will help for auditing of current practices and improve the standards of care.

Blood stained urine was the major complication observed in $60 \%$ of patients followed by difficulty in extraction of the baby in $48 \%$. The lower uterine segment tear and angle extension were observed in $12 \%$ of patients; PPH and Broad ligament hematoma were noted in one in each patient.

Mean duration of hospital stay is 2.28 days. Longer duration was 5 days as she underwent total abdominal hysterectomy due to extensive uterine tears during caesarean section.

All the babies had APGAR score of more than 7 at 5 minutes. Nearly $20 \%(n=5)$ of the babies were admitted to PBU, among them one required neonatal resuscitation at birth and two received IV antibiotics for possible sepsis. There were no maternal and neonatal mortality reported in this study.

\section{Key words}

Second stage caesarean section, Feto-maternal outcome, maternal complications, neonatal complications

\section{Introduction}

Worldwide nearly $10-20 \%$ of deliveries require intervention which is frequently cesarean section1. It is the most commonly performed major abdominal surgery in women all over the world. $(2,3)$

Caesarean sections are effective in saving maternal and infant lives, when they are performed for medically indicated reasons. If the caesarean section rates are higher than $10 \%$ they are not associated with reductions in maternal and newborn mortality rates. $(4,5)$

However, in the present context, caesarean section rates for non-obstetric indications are rising. It can be attributed mainly to increasing maternal age, increased number of multiple births, and higher rates of obesity among women. The fear of labour pain, fear of medical litigation and the belief of caesarean sections prevents trauma and damage to the pelvic floor and less traumatic to the baby are also considered as reasons for the caesarian section.

According to the Royal College of Obstetricians and Gynecologists audit figures, about $35 \%$ of caesareans for singleton pregnancies are performed following failure to progress in labour, of which a quarter occur at full cervical dilatation.(6)

Second stage of labor lasts between full cervical dilatation and the fetal delivery. In the past the duration of second stage of labor was limited to $<$ 2 hours. $(7,8)$ Recently it is extended up-to three hours with regional anaesthesia.(9)

When an intervention is needed in the second stage of labour to facilitate delivery of the fetus, it is usually in the form of assisted vaginal delivery.(10) However, a drop in the rates of operative vaginal

Corresponding author : Thirukumar M, Email: dr.thiru10@yahoo.com , iD https://orcid.org/0000-0001-8499-7175 
delivery has been noted with a corresponding rise in the cesarean deliveries during second stage of labor.(11)

A great deal of technical difficulty is faced during the second stage cesarean due to engagement of the fetal head and is the main reason for the associated increased maternal and fetal morbidity. $(12,13)$

Allen et al found that maternal operative trauma and perinatal asphyxia were significantly increased in women undergoing caesarean section at full cervical dilatation compared to caesarean section at less than full dilatation.(14)

Moreover, maternal morbidity may be higher when a caesarean section is done during the second stage of labour, as it is more difficult, especially in cases of malposition (such as in the occipitoposterior position), cephalopelvic disproportion (CPD) or after attempted instrumental delivery. The major maternal risks that lead to increased maternal morbidity and mortality during second stage caesarean sections are major haemorrhage, trauma to the surrounding structures (such as the bladder, bowel and urethra), lacerations of the lower segment and involvement of the broad ligament. The second stage caesareans increase the maternal risks such as extension tears of the uterine angle leading to broad ligament haematoma, major obstetric haemorrhage, longer hospital stay and greater risk of bladder trauma.(14) An increase in neonatal mortality and morbidity is mainly due to hypoxia and fetal trauma. $(15,16)$

\section{Methodology}

This is a prospective observational study of 25 cases of cesarean sections done in second stage of labour in Teaching Hospital Batticaloa, Sri Lanka from June-October 2019. This study was approved by Institutional ethics committee.

Data regarding the factors influencing maternal and perinatal outcomes during second stage caesarean sections and the associated maternal and perinatal morbidities were collected using a data sheet.

In this study, the onset of labor was defined as the initiation of regular painful uterine contractions. The second stage of labor was defined as the period of time from full cervical dilatation $(10 \mathrm{~cm})$ to delivery.

The following inclusion criteria were used: Singleton pregnancy irrespective of parity, Period of gestation of $>37$ weeks, Cephalic presentation and With/without previous LSCS. The medical conditions complicated pregnancies were excluded from the study.

\section{Results}

There were total 1912 deliveries during the study period. Among them 637 (30\%) cesarean sections were performed including the 25 cases $(3.9 \%)$ of the second stage LSCS.

The mean age of the patients who underwent cesarean in the second stage was 25.78 year. Among these 25 patients, $15(60 \%)$ were primi gravida and $10(40 \%)$ were multi gravida. The gestational age ranged between $38-40$ weeks in nearly $56 \%$ and above 40 weeks in $40 \%$ patients with only $4 \%$ less than 38 weeks (Table 1).

\section{Table 1 Maternal Characteristics}

\begin{tabular}{|l|c|c|}
\hline Parity & Number & Percentage \\
\hline Primi & 15 & $60 \%$ \\
\hline Multi & 10 & $40 \%$ \\
\hline & & \\
GESTATIONALAGE & & \\
\hline$<38$ weeks & 01 & $4 \%$ \\
\hline $38-40$ weeks & 14 & $56 \%$ \\
\hline$>40$ weeks & 10 & $40 \%$ \\
\hline
\end{tabular}

Most common fetal position was the occipito anterior position $(n=17,68 \%)$; Seven $(28 \%)$ were in occipito-posterior position. Only one (4\%) fetus was in occipito-transverse position. (Table 2)

Table 2 Fetal positions during caesarean section

\begin{tabular}{|l|c|c|}
\hline Position of foetus & Number & Percentage \\
\hline Occipito - anterior & 17 & $68 \%$ \\
\hline Occipito - posterior & 07 & $28 \%$ \\
\hline Occipito - tranverse & 01 & $4 \%$ \\
\hline
\end{tabular}

All the second stage cesarean sections were performed due to delayed second stage. No 
particular causes for the delay were noted in this study. Either vacuum or forceps were attempted and failed in $48 \%(n=13)$ of patients. Rest $52 \%(n-12)$, instrumental delivery was not attempted.

In three deliveries the operator attempted digital rotation to rotate the fetus from occipito-posterior to occipito-anterior position during section. Except in one case, all the other cases, experienced obstetrician was present and the caesarian section was supervised by them.

Blood stained urine was the major complication observed in $60 \%(n=15)$ of patients followed by difficulty in extraction of the baby in $48 \%(n=12)$ of deliveries. The lower uterine segment tear and angle extension were observed in $12 \%(n=3)$ of patients. Second stage cesareans are associated with increased risk of PPH but only one patient had PPH in our study. Broad ligament hematoma was also observed only in 1 patient.

Mean duration of hospital stay is 2.28 days. Longer duration was 5 days as she underwent total abdominal hysterectomy due to uterine tears during the surgery. There were no other complications happened to them during caesarian section and no maternal mortality in the present study (Table 3 ).

Table $3 \quad$ Maternal morbidities

\begin{tabular}{|l|c|c|}
\hline $\begin{array}{l}\text { Complication en- } \\
\text { countered }\end{array}$ & $\begin{array}{l}\text { Number } \\
\text { of patients }\end{array}$ & $\begin{array}{l}\text { Percent- } \\
\text { age }\end{array}$ \\
\hline $\begin{array}{l}\text { LUS tear, angle } \\
\text { extension }\end{array}$ & 03 & $12 \%$ \\
\hline $\begin{array}{l}\text { Broad ligament } \\
\text { hematoma }\end{array}$ & 01 & $4 \%$ \\
\hline PPH & 01 & $4 \%$ \\
\hline Extraction difficulty & 12 & $48 \%$ \\
\hline Bladder damage & 00 & 00 \\
\hline Blood stained urine & 15 & $60 \%$ \\
\hline Post-operative fever & 00 & 00 \\
\hline Wound sepsis & 00 & 00 \\
\hline Ureteric damage & 01 & $4 \%$ \\
\hline
\end{tabular}

Among the 26 babies born 12 (48\%) were female and $13(52 \%)$ were male babies. The majority of the babies' weights were within the normal range (2500g-3500). Number of babies with birth weight $>3.5 \mathrm{~kg}$ is $4(16 \%)$; among those $5(20 \%)$ babies had neonatal complications following caesarian section in second stage labour. Meconium stained amniotic fluid was present in $28 \%$ of the cases $(n=7)$ (Table 4).

All the babies hadAPGAR score of more than 7 at 5 minutes. $20 \%(n=5)$ of babies were admitted to PBU, among them one required neonatal resuscitation at birth and two received IV antibiotics. There was no neonatal mortality in the present study.

\section{Table 4 Neonatal outcomes}

\begin{tabular}{|l|c|c|}
\hline Sex of baby & Number & Percentage \\
\hline Male & 13 & $52 \%$ \\
\hline Female & 12 & $48 \%$ \\
\hline $\begin{array}{l}\text { Birth weight of } \\
\text { Baby (g) }\end{array}$ & 01 & $4 \%$ \\
\hline $1500-2500$ & 20 & $80 \%$ \\
\hline $2500-3500$ & 04 & $16 \%$ \\
\hline$>3500$ & 07 & $28 \%$ \\
\hline $\begin{array}{l}\text { Meconium stained } \\
\text { liquor }\end{array}$ & 18 & $72 \%$ \\
\hline Yes & 05 & $20 \%$ \\
\hline No & 20 & $80 \%$ \\
\hline $\begin{array}{l}\text { Any neonatal com- } \\
\text { plication }\end{array}$ & & \\
\hline Yes & & \\
\hline No & & \\
\hline
\end{tabular}

\section{Discussion}

Delayed second stage was the only indication for the caesarian section in this study. Specific causes for the delay were not studied and no fetal distress was noted. But the other related studies show, deep transverse arrest was the most common indication $(38.46 \%)$ for cesarean section in second stage of labor.(17) The study conducted by Jonna Malathi and Venigalla Sunita had the rate of second stage cesarean section $4.1 \%$. In the same study deep, transverse arrest was the second most common indication $(22 \%)$ for cesarean section in the second stage.

In this study, the mean age of patients who underwent cesarean in the second stage was 25.78 years. Among these, the percentage of primi gravida $(57.6 \%)$ is slightly higher than multi gravida (42.4\%). These results are somewhat similar to the study conducted in rural medical college, Adichunchangiri institute of medical sciences (primi was 61.53\%) and study by Shahla Baloch et al.(10) The higher frequency 
of second stage cesareans in primi gravidas could be cephalopelvic disproportion, rigid perineum and lack of experience of previous labour.(17)

In this study, the gestational age ranged between $38-40$ weeks in nearly $56 \%$ and above 40 weeks in $40 \%$ patients with only $4 \%$ less than 38 weeks.

In a study on frequency of second stage interventions and it's outcome in relation with instrumental vaginal delivery by Shahla Baloch et al.10 48\% were attempted either vacuum or forceps and failed before deciding on caesarean section. Probably due to less skill in operative vaginal delivery and concerns over maternal and neonatal morbidity associated with difficult or failed instrumental delivery.(18)

Blood stained urine $(60 \%)$ the most common maternal complication seen in the present study, probably due to bladder congestion, oedema and obstruction. This may also be due to unrecognized bladder mucosal damage without complete bladder wall damage. It is similar (76.2\%) to the study done in the department of Obstetrics and Gynaecology in a tertiary care Hospital, Bangalore, Karnataka, India. It is followed by difficulty in extraction of the baby $(\mathrm{n}=12,48 \%)$. Other complications are rare occurrence in our study though they were some of the common problems in other studies such as study done by Malathi and Sunita.(17) and Shahla B.(10)

Mean duration of hospital stay in our present study is 2.28 days. This is much lesser than the study done by Jyoti Jayaram et al. In their study, longer hospital stay is one of the complications and the mean duration being 8.9 days.(24)

The cesarean section performed in second stage of labor was technically difficult because fetal head was engaged in the pelvis, uterine muscles were thin $\&$ tense and the identification of the bladder and lower segment was difficult.

Neonatal morbidity was not much significant in the present study. The majority of the babies weigh between $2.5 \mathrm{~kg}$ to $3.5 \mathrm{~kg}$. Meconium stained amniotic fluid was present in $28 \%$ cases. There were controversies regarding the fetal outcome in the cases of caesarean sections in second stage of labour. Study by Asicioglu.(19) Malathi.(17) etc. had proved adverse prognostic impact on foetal outcome. But many studies like Allen et al,
Alexander.(20), Selo-Ojeme.(21) etc. including the current study failed to demonstrate an increased foetal complication.

In this study. five neonates (20\%) were admitted to the neonatal unit. Among them only one neonate required neonatal resuscitation as the baby was found floppy at birth and two were given with prophylactic antibiotics. This is less when compared to the studies by Sandya MR et al.(22) and by Unterscheider J.(23) where the PBU admission rate was above $30 \%$.

This study showed that either vacuum or forceps were attempted and failed in $48 \%(n=13)$ of patients. This figure is similar to the audit results of the Royal College of Obstetricians and Gynecologists in UK. It showed that about $35 \%$ of caesareans for singleton pregnancies were performed because of failure to progress in labour, of which a quarter occur at full cervical dilatation. In 55\% of these cases no attempt was made to achieve a vaginal birth with either forceps or ventouse. In those births where instrumental delivery was attempted, the audit noted a "failed" rate of $35 \%$ for ventouse and $2 \%$ for forceps.(6)

There is a worrying rise in the overall rate of caesarean section at full dilatation. Better training in instrumental delivery may reduce rates of second stage caesarean section. Audit of the second stage caesarean section rate is a useful measure of clinical standards. Strategies for improved care include increased consultant assessment of the patient, more supervision and training of junior obstetric staff by consultant to ensure safe intrapartum care.

\section{Conclusion}

The choice between a difficult caesarean section in second stage of labour and instrumental delivery is challenging. Although second stage caesarean section is sometimes appropriate, many could be prevented by the attendance of a more skilled obstetrician. The involvement of senior obstetrician is desired during the process of decision making. Better training in instrumental delivery may reduce rates of second caesarian sections.

\section{Acknowledgement}

I would like thank to the director and obstetric team members of teaching hospital Batticaloa for granting permission to collect the data. 


\section{References}

1. William obstetrics Obstetrics .24 th edition 2014 by McGraw-Hill Education; Operative vaginal delivery 2014;574-586

2. Murray S F, Pradenas F S,.Health sector reform and rise of cesarean birth in Chile. The Lancet;1997-64.

3. Pai M et al. A high rate of cesarean sections in an affluent section of Chennai: is it cause for concern? National Med J Ind 1999(12):156-58.

4. WHO Statement on Caesarean Section Rates.

5. Ye J, Betran AP, Vela MG, Souza JP, Zhang J. Searching for the Optimal Rate of Medically Necessary Cesarean Delivery. Birth. 2014;41(3):237-43.

6. Thomas J, Paranjothy S, Royal College of Obstetricians and Gynaecologists Clinical Effectiveness Support Unit. The national sentinel caesarean section audit report. London: RCOG Press, 2001.

7. Moodley J, Devjee J, Khedun S and Esterhuizen $\mathrm{T}$. Second stage primary cesarean deliveries: are maternal complications increased? South African family Practice.2009:328-31.

8. Kilpatrik S J, Laros R Characteristics of normal labor. J Obstet Gynaecol 1989;(74):857.

9. Myles T D, Santolaya J. Maternal and neonatal outcomes in patients with a prolonged stage of labor. J obstet Gynaecol 2003;(102):52-8.

10. Baloch S ,Khaskheli M, Khushk I A, Sheeba A. Frequency of second stage intervention and it's outcome in relation with instrumental vaginal delivery versus section. J Ayub Coll Abbotabad 2008;20(1):87-90.

11. Black C, Kaye JA, Jick H. Cesarean delivery in the United Kingdom: time trends in the General Practice Research Database. Obstet Gynecol 2005; 106:151-5.

12. Murphy D J et al. Early maternal and neonatal morbidity associated with operative delivery in the second stage of labor: a cohort study. Lancet 2001; 358:1203-7.

13. O B et al. Delivery of the impacted head of the fetus at cesarean section after prolonged obstructed labor: a randomized comparative study of two methods. J obstet Gynecol 2002; 22:375-8.
14. Allen VM, O'Connell CM, Baskett TF. Maternal and Perinatal morbidity of caesarean delivery at full cervical dilatation compared with caesarean delivery in the first stage of labour. BJOG. 2005; 112:986-90.

15. Seal S L et al. Outcome in second - vs first -stage cesarean delivery in teaching institution in Estern India. Am J Perinat 2010:507-12.

16. Winovitch K C, Wing DA, Lagrew D C, Chung J H. The risk of acute neonatal morbidities in the delivery room after primary cesarean at term: influence of labor and stage. Am J of Pernat 2009:545-51.

17. Malathi J,Sunita V. Comparison of obstetric outcome between first and second stage cesarean sections in rural tertiary hospital.Int J Pharm biomed Reas 2012:222-25.

18. Stavrou EP, Ford JB, Shand AW (2011). Epidemiology and trends for caesarean section births in New South Wales, Australia: a populationbased study. BMC Pregnancy Childbirth. 11: 8.

19. Asicioglu O et al. Second stage vs first-stage cesarean delivery: comparison of maternal and perinatal outcomes. J Obstet and Gynecol;2014:1-7.

20. Alexander JM, Leveno KJ, Rouse DJ (2007). Comparison of maternal and infant outcomes from primary caesarean delivery during the second compared with the first stage of labor. Obstet\& Gynecol. 109 (4): 917921.

21. Selo-Ojeme D, Sathiyathasan S, Fayyaz M (2008). Caesarean delivery at full cervical dilatation versus caesarean delivery during the second compared with the first stage of labor. Obstet. Gynaecol. 109 (4): 917921.

22. Sandya MR, Shirley George and Lissy Varghese (2018). Fetomaternal outcome in second stage Caesarean section. Int. J. Obstet. Gynaecol. ISSN 2326-7234 Vol. 6 (2), pp. 207-210, February, 2018.

23. Unterscheider J, McMenamin M, Cullinane F (2011). Rising rates of caesarean deliveries at full cervical dilatation: a concerning trend. Eur J Obstet. Gynecol. Reprod Biol. 157 (2): 141-144.

24. Jyoti Jayaram et al. (2016). Fetomaternal Outcome in Cesarean Sections Done in Second Stage of Labor. Indian Journal of Obstetrics and Gynaecology Research 2016;3(1):51-54. 